\title{
Molecular cytogenetic characterization of parental genomes in the partial amphidiploid Triticum aestivum $\times$ Thinopyrum ponticum
}

\author{
Ana Christina Brasileiro-Vidal ${ }^{1}$, Angeles Cuadrado ${ }^{2}$, Sandra P. Brammer ${ }^{3}$, Ana Maria Benko-Iseppon ${ }^{4}$ \\ and Marcelo Guerra ${ }^{1}$ \\ ${ }^{1}$ Universidade Federal de Pernambuco, Departamento de Botânica, Recife, Pernambuco, Brazil. \\ ${ }^{2}$ Universidad de Alcalá, Departamento de Biología Celular y Genética, Alcalá de Henares, Madrid, Spain. \\ ${ }^{3}$ Empresa Brasileira de Pesquisa Agropecuária, Embrapa Trigo, Passo Fundo, Rio Grande do Sul, Brazil. \\ ${ }^{4}$ Universidade Federal de Pernambuco, Departamento de Genética, Recife, Pernambuco, Brazil.
}

\begin{abstract}
The wheat line PF 839197 and six hybrid derivatives from a cross between PF 839197 and Thinopyrum ponticum were cytologically characterized by fluorescent in situ hybridization (FISH). Probes for the $5 \mathrm{~S}$ and $45 \mathrm{~S}$ rDNA genes (pTa794 and pTa71, respectively), a highly repetitive rye sequence (pSc119.2), the synthetic oligonucleotide (AAG) and total genomic DNA from Th. ponticum and rye were used. In the wheat line, a $1 \mathrm{RS} .1 \mathrm{BL}$ translocation was revealed by the labeling patterns produced with pSc119.2 and (AAG) ${ }_{5}$, and confirmed by genomic in situ hybridization (GISH) using rye genomic DNA as a probe. Analyses of partial amphiploids confirmed previous results indicating mitotic instability, with a tendency to stabilize at $2 n=42$ or 56 . GISH with Th. ponticum genomic DNA showed that in one hybrid derivative, with lower chromosome numbers $(2 n=42-45)$, chromosomes were not labeled, whereas in the hybrids with $2 n=48-56$ up to 14 chromosomes were labeled. These data suggest that the original chromosome set of these hybrids was $2 n=56$, and that chromosomes from both genomes were lost by mitotic instability. FISH using the rDNA probes and GISH with Thinopyrum genomic DNA suggested that cells with $2 \mathrm{n}=56$ contained an entire wheat genome plus two monoploid chromosome sets of Th. ponticum.
\end{abstract}

Key words: wheat, Thinopyrum ponticum, rye, hybrid derivatives, GISH.

Received: Febuary 13, 2004; Accepted: July 20, 2004.

\section{Introduction}

Thinopyrum ponticum (Podp.) Barkworth \& D. R. Dewey has been extensively used in wheat breeding programs as a source for new genes, such as the resistance genes against leaf and stem rust (McIntosh et al., 1998). This species is decaploid $(2 \mathrm{n}=10 \mathrm{x}=70)$ including the genomes $\mathrm{JJJJ}^{\mathrm{s}} \mathrm{J}^{\mathrm{s}}$, with $\mathrm{J}$ and $\mathrm{J}^{\mathrm{s}}$ proximally related (Chen et al., 1998a). As other Triticeae species, Th. ponticum has a symmetric karyotype, hindering the identification of individual chromosomes. Furthermore, no banding pattern allowing the identification of each homoeology group was reported for this species.

In a recent attempt to better characterize the karyotype of Th. ponticum, Brasileiro-Vidal et al. (2003) reported the presence of $205 \mathrm{~S}$ rDNA and $1745 \mathrm{~S}$ rDNA sites, in addition to other sites of satellite DNA sequences.

Send correspondence to Ana Christina Brasileiro-Vidal. Universidade Federal de Pernambuco, Cidade Universitária, Departamento de Botânica, CCB, 50670-420 Recife, Pernambuco, Brazil. E-mail: brasileiro_vidal@ hotmail.com.
The 45S rDNA sites were terminally located on the short arms of the $5 \mathrm{~S}$ rDNA bearing chromosomes. Considering the basic number $\mathrm{x}=7$, the decaploid nature and the chromosome morphology of this species, the 17 linked 5S-45S rDNA loci and the three single 5S rDNA loci seem to be restricted to two homeologous groups.

Many partial wheat $\times$ Thinopyrum amphiploids with $2 \mathrm{n}=8 \mathrm{x}=56$ have been produced by backcrossing the wheat $\times$ Thinopyrum hybrid with wheat. These amphiploids usually contain a complete wheat complement (42 chromosomes) and two monoploid Thinopyrum complements (14 chromosomes), but sometimes there are some substitutions of wheat chromosomes by Thinopyrum chromosomes or the opposite (Fedak et al., 2000). The genomic composition of these amphiploids has been analyzed by genomic in situ hybridization (GISH) using genomic DNA of several species as probes (Chen et al., 1998b; Cai et al., 2001). Thus, the $\mathrm{J}$ and $\mathrm{J}^{\mathrm{s}}$ genomes have been distinguished by the presence of sequences from the St genome (Pseudoroegneria strigosa, StSt), mainly in the centromeric regions of $\mathrm{J}^{\mathrm{s}}$ chromosomes (Chen et al., 1998a). 
Other probes, such as synthetic oligonucleotides and the repetitive sequence $\mathrm{pSc} 119.2$ isolated from rye, have also been very useful for genomic characterization of interspecific hybrids (Cuadrado and Schwarzacher, 1998). In wheat, fluorescent in situ hybridization (FISH) with $(\mathrm{AAG})_{5}$ or with a probe rich in GAA repeats results in a banding pattern similar to the $\mathrm{N}$ banding (Pedersen and Langridge, 1997; Cuadrado et al., 2000). As a result, the seven pairs of the B genome are easily recognizable, as well as some $\mathrm{A}$ and $\mathrm{D}$ chromosomes in intergeneric hybrids (Cuadrado and Schwarzacher, 1998).

The research unit "Embrapa Trigo", located in Passo Fundo (state of Rio Grande do Sul, Brazil), has developed a program to generate introgressions of Th. ponticum genes, mainly for leaf and stem rust resistance, in local wheat cultivars. Six accessions obtained from the wheat $\times T h$. ponticum hybrid backcrossed twice and self-fertilized for five to seven generations were analyzed for chromosome number and DNA content. The results revealed a chromosome number variation generated at least partially by mitotic instability (Brasileiro-Vidal, 2003). Five of these accessions revealed wide intra-individual variation, with higher chromosome numbers (around $2 \mathrm{n}=c a$. 56), while a single accession showed a more or less stable chromosome number, $2 n=42$ being the most frequent one.

In the present work, a combination of cytological methods, GISH and FISH, was used to characterize the genomic composition of the six above mentioned accessions and of the wheat line PF 839197 used as a parent in the first cross. The cultivar 'Alondra' is one of the ancestral lines of this wheat line and putatively carries a wheat-rye translocation on the short arm of chromosome 1, designated as 1BL.1RS (Schlegel, 2003). However, it is not known if the rye short arm was transmitted to the progeny and if it is still present in PF839197.

\section{Materials and Methods}

\section{Plant material and chromosome preparation}

The genotypes analyzed included the wheat line PF 839197 and six wheat-Th. ponticum partial amphiploids. The latter were produced by crossing line PF 839197 with the accession PF Ag. el. 84001 of Th. ponticum. The hybrid was backcrossed twice with the wheat cultivar 'CEP 19' (PF 839197/PF Ag. el. 84001//2*‘CEP 19'). Three accessions (PF 984501, PF 984504, PF 984506) were obtained by selfing for seven generations $\left(\mathrm{BC}_{2} \mathrm{~F}_{7}\right)$, and other three accessions (PF 984901A, PF 984902, PF 984903A) were derived from selfing for five generations $\left(\mathrm{BC}_{2} \mathrm{~F}_{5}\right)$ followed by haplodiploidization, as described by Laurie and Bennett (1986; 1989), modified by Suenaga and Nakajima (1989) and Inagaki and Tahir (1990). Haplodiploidization was achieved by crossing individuals of the $\mathrm{BC}_{2} \mathrm{~F}_{5}$ generation to maize plants. The resulting hybrid lost all maize chromosomes by somatic elimination, whereas the entire haploid chromosome set of the original hybrid derivative was maintained. Chromosome doubling was performed when the haploid plants reached the 4-5 tiller stage, using a solution containing $0.25 \%$ colchicine and $10 \%$ dimethyl sulfoxide for 3-4 hours (Brammer, 2000). The described material is part of the wheat breeding program of the Brazilian Agricultural Research Corporation (Embrapa Trigo, Passo Fundo, RS, Brazil).

Root tips were pretreated in ice-cold water $\left(\mathrm{ca} .0{ }^{\circ} \mathrm{C}\right)$ for $24 \mathrm{~h}$, fixed in ethanol: acetic acid $(3: 1, \mathrm{v} / \mathrm{v})$, and stored at $-20{ }^{\circ} \mathrm{C}$. The material was digested in an enzyme mixture containing 2\%(w/v) cellulase (Onozuka R10) and 20\% $(\mathrm{v} / \mathrm{v})$ pectinase (Sigma) for $1.5 \mathrm{~h}$ at $37^{\circ} \mathrm{C}$. Root tips were squashed in a drop of $45 \%$ acetic acid and frozen in liquid nitrogen.

\section{DNA probes and labeling}

Six DNA probes were used for in situ hybridization: (1) total genomic DNA of Th. ponticum; (2) total genomic DNA of Secale cereale; (3) clone pTa71, containing the 18S-5.8S-26S rDNA repeat unit from T. aestivum (Gerlach and Bedbrook, 1979); (4) clone pTa794, that includes the complete 5S rRNA gene unit from T. aestivum (Gerlach and Dyer, 1980); (5) clone pSc119.2, containing the 120-pb repeat unit of a tandemly arranged DNA family derived from S. cereale (McIntyre et al., 1990), and (6) the synthetic oligonucleotide (AAG) 5 .

Probes were labeled with digoxigenin-11-dUTP (Roche), biotin-11-dUTP (Sigma) or rhodamine-5-dUTP (Sigma) using nick translation (genomic DNA and pTa71), random primer (synthetic oligonucleotide) or the polymerase chain reaction (pTa794 and pSc119.2) with universal forward and reverse primers, at an annealing temperature of $55^{\circ} \mathrm{C}$. In the GISH analyses, the genomic DNA probes of $T h$. ponticum and $S$. cereale were added to the hybridization mixture at the ratios of 1:40 and 1:10, respectively, with non-labeled blocking genomic wheat DNA.

\section{In situ hybridization}

In situ hybridization was performed essentially as described by Heslop-Harrison et al. (1991), at 85\% stringency. Digoxigenin-labeled probes were detected using anti-digoxigenin-fluorescein isotiocyanate (FITC) conjugate (Boehringer), while biotin-labeled probes were detected using avidin-rhodamine or avidin-FITC conjugate (Vector). All preparations were counterstained with $2 \mu \mathrm{g} / \mathrm{mL}$ 4', 6-diamidino-2-phenylindole (DAPI) and mounted in Vectashield H-1000 (Vector). The best cells were captured with a Leica DMLB microscope equipped with a Cohu CCD camera and the Leica QWin software or photographed on Fuji Super G or Kodak Ultra ASA 400 color films, and scanned at $300 \mathrm{dpi}$. The images were optimized for best contrast and brightness with Adobe Photoshop 6.0. 


\section{Results}

The hybridization patterns obtained with the probes pTa794, pTa71, pSc119.2 and (AAG) $)_{5}$ on the cultivar PF 839197 were similar to that previously described for 'Chinese Spring' (Mukai et al., 1990; 1991; Cuadrado and Jouve, 1994; Cuadrado et al., 2000), except for the short arm of chromosome 1B, which exhibited a different pattern

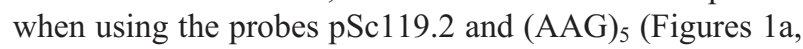
b). With these two probes, the chromosome arm 1BS showed the typical pattern of rye 1RS: probe pSc119.2 hybridized to both terminal and intercalary positions along this arm, with no hybridization signal with the $(A A G)_{5}$ oligonucleotide probe. Using GISH with rye genomic DNA as probe, two chromosome arms of rye were observed, confirming the translocation 1BL.1RS in this wheat line (Figure 1c).

A second GISH experiment using genomic DNA of Th. ponticum as probe in one of the $\mathrm{BC}_{2} \mathrm{~F}_{5}$ lines ( $\mathrm{PF}$ 984902), with $2 n=42$ to $2 n=45$, indicated the absence of fragments or whole chromosomes of Thinopyrum. On the other hand, in five other accessions analyzed (two $\mathrm{BC}_{2} \mathrm{~F}_{5}$ and three $\mathrm{BC}_{2} \mathrm{~F}_{7}$ ), the same probe revealed a maximum of 14 out of a total of 56 chromosomes, with uniform labeling along the whole chromosomes (Figure 1d). Due to the mi-
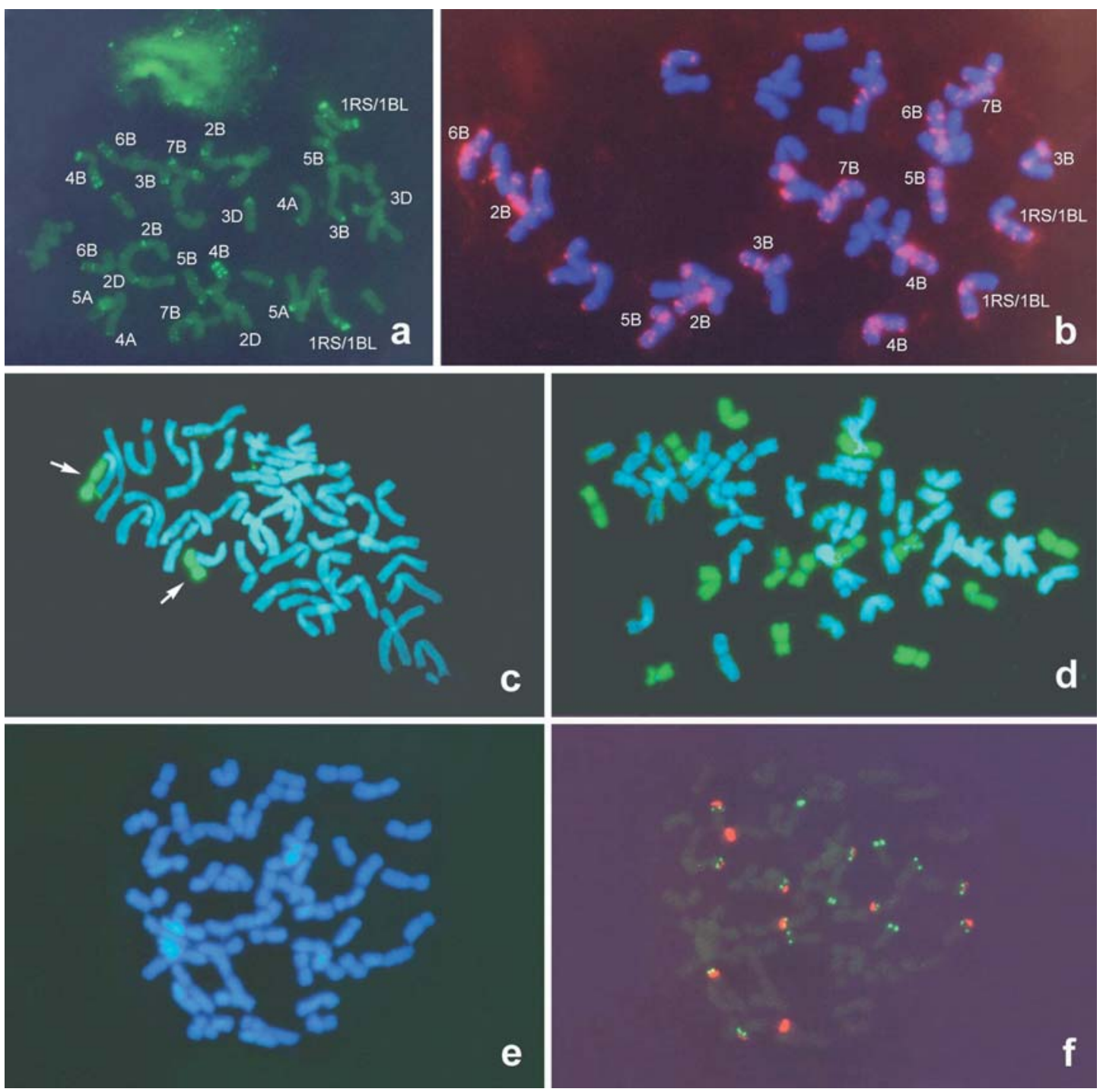

Figure 1 - In situ hybridization of mitotic metaphases of the wheat line PF 839197 and hybrid accessions derived from PF 839197 Thinopyrum ponticum. (a) Complete metaphase of the wheat line PF 839197 with $2 n=42$, revealing the distribution of pSc119.2 (green). Chromosomes were identified according to the distribution pattern of pSC119.2, as described by Cuadrado and Jouve (1994). (b) A metaphase of the same line after hybridization with (AAG) oligonucleotide (pink). Chromosomes are counterstained in blue (DAPI). The B genome chromosomes of wheat were identified according to Cuadrado et al. (2000). (c) GISH in PF 839197, confirming the 1RS translocation (green) to a wheat 1BL arm. Arrows indicate the pair of translocated 1RS.1BL chromosomes. (d) GISH of the amphidiploid PF 984506 with $2 n=56$ and 14 Th. ponticum chromosomes (green). (e, f) Metaphase of the same accession with $2 \mathrm{n}=56$, stained with DAPI (e) and showing sixteen 5S rDNA sites (green) and twelve 45S rDNA sites (red) (f). 
totic instability, $59 \%$ of the 44 analyzed cells showed lower chromosome numbers $(2 \mathrm{n}=48$ to $2 \mathrm{n}=55)$, with 11 to 14 labeled chromosomes. No wheat-Thinopyrum translocation could be observed.

In situ hybridization with rDNA probes in cells with $2 \mathrm{n}=56$ revealed six chromosomes bearing only $5 \mathrm{~S}$ rDNA sites (probably 5A, 5B and 1D), two with only 45S rDNA sites (probably $6 \mathrm{~B}$ ), and six with both $5 \mathrm{~S}$ and $45 \mathrm{~S}$ rDNA sites (probably 1A, 1B and 5D), as previously observed in wheat (Mukai et al., 1990; 1991), plus four other chromosomes bearing $5 \mathrm{~S}$ and $45 \mathrm{~S}$ rDNA (Figures $1 \mathrm{e}, \mathrm{f}$ ).

\section{Discussion}

The presence of the rye $1 \mathrm{RS}$ chromosome arm in wheat cultivars affects their performance positively and also confers resistance against insect and pathogen attacks (Caver and Rayburn, 1994). Many wheat cultivars carry the $1 \mathrm{RS}$ arm, but in most cases one group 1 chromosome has its original arm substituted by 1RS (Berzonsky and Francki, 1999). The present in situ evaluation using rye genomic DNA and the probes pSc119.2 and (AAG) $)_{5}$ revealed the presence of the 1BL.1RS translocation in the line $\mathrm{PF}$ 839197, probably derived from the cultivar 'Alondra'.

The mitotic instability, reported by Brasileiro-Vidal (2003) in the same accessions, led to chromosome numbers around $2 \mathrm{n}=42$ in the double-haploid PF $984902\left(\mathrm{BC}_{2} \mathrm{~F}_{5}\right.$ line) and around $2 n=56$ in the remaining partial amphidiploids. In the first case $(2 \mathrm{n}=42)$, no Th. ponticum chromosomes were found in the accession mentioned. Likewise, Cai et al. (1998b) also observed that the Th. ponticum genome had been completely eliminated from some $\mathrm{BC}_{1} \mathrm{~F}_{2}$ individuals of the cross wheat $\times$ Thinopyrum. In the remaining accessions, the cells with $2 \mathrm{n}=56$ had always 42 wheat and 14 Thinopyrum chromosomes, whereas cells with $2 \mathrm{n}=48$ to $2 \mathrm{n}=55$ had a variable number of both genomes. These data suggest that the zygotic chromosome number of these individuals is likely to be $2 n=56$, and that the mitotic instability has produced chimerical tissues with different chromosome numbers and an uneven proportion of wheat and Thinopyrum genomes. Brasileiro-Vidal (2003) observed that this mitotic instability was already present in the cultivar used as recurrent wheat parent (' $\mathrm{CEP}$ 19 '). The same phenomenon was previously reported for other Brazilian wheat cultivars (Guerra and Moraes-Fernandes, 1977), and it was probably enhanced by the hybrid condition. Influence of the parental genotypes on chromosome elimination has also been observed in wide-cross hybrids, and differential elimination seems to be dependent on the ordered spatial arrangement of chromosomes throughout the cell cycle (see Heslop-Harrison and Schwarzacher, 1993).

The biparental chromosome losses found in the present work are in contrast to previous reports for wide-cross hybrids, such as barley $\times$ Hordeum bulbosum, wheat $\times$ maize, wheat $\times$ sorghum, and barley $\times$ maize, in which the mitotic chromosome elimination was always uniparental (Laurie and Bennett, 1988; Finch, 1983). However, hybrids of wheat with closely related genera, like Agropyron and Thinopyrum, tend to be rather stable (Chen et al., 1992; Cai et al., 1998b). Therefore, if mitotic instability is present in such hybrids, it should affect both parental genomes.

Partial amphidiploids of wheat $\times$ Th. ponticum with $2 n=56$ chromosomes were reported before, but their genomic composition was variable. In line AT 3425, for example, seven Th. ponticum chromosome pairs could be identified, as well as three additional pairs of translocated wheat-Thinopyrum and 18 pairs of wheat chromosomes (Cai et al., 1998a). In the line Agrotana, a total of 40 wheat and 16 Th. ponticum chromosomes were observed (Chen et al., 1995). Line 784 had a similar composition as observed in the present work, with 42 wheat and $14 \mathrm{Th}$. ponticum chromosomes (Zhang et al., 1996).

Chen et al. (1995) suggested that Agrotana could be derived from the fusion of reduced or non-reduced gametes, followed by the loss of chromosomes at the beginning of the subsequent backcrosses and selfing procedures. Chen et al. (1999b) suggested that the genome of the donating species in partial amphidiploids of wheat $\times$ Thinopyrum may not necessarily be an intact genome of the donor species, but a synthetic genome combining chromosomes from both species. The alien genomic composition of the partial amphidiploid TAF46, for example, is a synthetic genome containing seven pairs of homoeologous chromosomes, that works as a single unit in terms of stability and transmission of its chromosomes through the gametes. The alien genome, once formed, is quite stable. The data presented here seem to confirm this observation, since five of the six accessions maintained ca. 14 chromosomes of Th. ponticum, and only one accession was entirely depleted of Thinopyrum chromosomes.

Partial amphidiploids of this type, with $2 n=56$, have normally a regular meiosis with high frequencies of bivalent and low multivalent formation (Fedak et al., 2000). In Agrotana, for example, eight Thinopyrum-Thinopyrum bivalents, 16-20 wheat-wheat bivalents and no allosyndetic pairing were observed. This meiotic behavior could guarantee high fertility and chromosome stability (Chen et al., 1995). In the cells with $2 n=56$ analyzed here, the detection of four chromosomes with $5 \mathrm{~S}$ and $45 \mathrm{~S}$ rDNA sites, in similar positions to those observed in Th. ponticum (Brasileiro-Vidal et al., 2003), suggests that the $14 \mathrm{Th}$. ponticum chromosomes represent two monoploid complements, since each monoploid chromosome set of $T h$. ponticum has two $5 \mathrm{~S}$ and two $45 \mathrm{~S}$ rDNA sites (Brasileiro-Vidal et al., 2003). It was not possible to recognize to which genome $\left(\mathrm{J}\right.$ or $\left.\mathrm{J}^{\mathrm{s}}\right)$ these chromosomes belonged. Partial amphidiploids do not have necessarily the same chromosome set as the introgressed species; there- 
fore, they can be composed of different rates of $\mathrm{J}$ and $\mathrm{J}^{\mathrm{s}}$ chromosomes. Zhang et al. (1996) suggested that, of the 14 Th. ponticum chromosomes of line 784 , six pairs came from the $\mathrm{J}^{\mathrm{s}}$ and one from the $\mathrm{J}$ genome. On the other hand, Chen et al. (1998b; 1999a) identified eight $\mathrm{J}$ and eight $\mathrm{J}^{\mathrm{s}}$ chromosomes in Agrotana.

If the 14 Th. ponticum chromosomes reported here in cells with $2 n=56$ form seven bivalent pairs in meiosis, they could be maintained by selfing with a stable meiotic transmission. The mitotic instability of these accessions generates a mosaic of cells with chromosome numbers different from $2 n=56$, but it does not seem to affect the production of gametes to the next generation. A GISH evaluation of meiotic cells may reveal the maintenance mechanism of such amphidiploids even in the presence of the observed mitotic instability.

\section{Acknowledgements}

The authors thank the Brazilian agencies Conselho Nacional de Desenvolvimento Científico e Tecnológico (CNPq), Programa de Apoio a Núcleos de Excelência (PRONEX-CNPq) and Fundação de Amparo à Ciência e Tecnologia do Estado de Pernambuco (FACEPE) for fellowships and financial support. The authors are very grateful to Dilma Angra for helpful suggestions.

\section{References}

Berzonsky WA and Francki MG (1999) Biochemical, molecular, and cytogenetic technologies for characterizing 1RS in wheat: A review. Euphytica 108:1-19.

Brammer SP (2000) Mapeamento de genes de resistência parcial à ferrugem da folha em cultivares brasileiras de trigo (Triticum aestivum L. em Thell). PhD Thesis, Universidade Federal do Rio Grande do Sul, Porto Alegre.

Brasileiro-Vidal AC (2003) Caracterização cromossômica de híbridos intergenéricos de trigo (Triticum aestivum $\mathrm{x}$ Thinopyrum ponticum) com diferentes combinações genômicas. PhD Thesis, Universidade Federal de Pernambuco, Recife.

Brasileiro-Vidal AC, Cuadrado A, Brammer SP, Zanatta ACA, Prestes AM, Moraes-Fernandes MIB and Guerra M (2003) Chromosome characterization in Thinopyrum ponticum (Triticeae, Poaceae) using in situ hybridization with different DNA sequences. Gen Mol Biol 26:505-510.

Cai X, Jones SS and Murray TD (1998a) Molecular cytogenetic characterization of Thinopyrum and wheat-Thinopyrum translocated chromosomes in a wheat-Thinopyrum amphiploid. Chromosome Res 6:183-189.

Cai X, Murray TD and Jones SS (1998b) Identification and manipulation of Thinopyrum ponticum chromatin conferring resistance to Cephalosporium stripe disease and a perennial habit in wheat. Proc $9^{\text {th }}$ Int Wheat Genet Symp, University of Saskatchewan, Saskatoon, pp 29-31.

Cai X, Jones SS and Murray TD (2001) Molecular cytogenetic characterization of Thinopyrum genomes conferring perennial growth habit in wheat-Thinopyrum amphiploids. Plant Breed 120:21-26.
Caver BF and Rayburn AL (1994) Comparison of related wheat stocks possessing 1B or 1RS.1BL chromosomes: Agronomic performance. Crop Sci 34:1505-1510.

Chen Q, Jahier J and Cauderon Y (1992) Production and cytogenetic analysis of $\mathrm{BC}_{1}, \mathrm{BC}_{2}$, and $\mathrm{BC}_{3}$ progenies of an intergeneric hybrid between Triticum aestivum (L.) Thell. and tetraploid Agropyron cristatum (L.) Gaertn. Theor Appl Genet 84:698-703.

Chen Q, Conner RL and Laroche A (1995) Identification of the parental chromosomes of the wheat-alien amphiploid Agrotana by genomic in situ hybridization. Genome 38:1163-1169.

Chen Q, Conner RL, Laroche A and Thomas JB (1998a) Genome analysis of Thinopyrum intermedium and Thinopyrum ponticum using genomic in situ hybridization. Genome 41:580-586.

Chen Q, Conner RL, Laroche A and Thomas JB (1998b) Molecular characterization of the genome composition of partial amphiploids derived from Triticum aestivum Thinopyrum ponticum and Triticum aestivum Thinopyrum intermedium as sources of resistance to wheat streak mosaic virus and its vector, Aceria tosichella. Theor Appl Genet 97:1-8.

Chen Q, Conner RL, Laroche A, Fedak G and Thomas JB (1999a) Genomic origins of Thinopyrum chromosomes specifying resistance to wheat streak mosaic virus and its vector, Aceria tosichella. Genome 42:289-295.

Chen Q, Conner RL, Laroche A, Armstrong KC and Fedak G (1999b) Genomic in situ hybridization analysis of Thinopyrum chromatin in a wheat-Th. intermedium partial amphiploid and six derived chromosome addition lines. Genome 42:1217-1223.

Cuadrado A and Jouve N (1994) Mapping and organization of highly-repeated DNA sequences by means of simultaneous and sequential FISH and C-banding in 6x-triticale. Chom Res 2:331-338.

Cuadrado A and Schwarzacher T (1998) The chromosomal organization of simple sequence repeats in wheat and rye genomes. Chromosoma 107:587-594.

Cuadrado A, Schwarzacher T and Jouve N (2000) Identification of different chromatin classes in wheat using in situ hybridization with simple sequence repeat oligonucleotides. Theor Appl Genet 101:711-717.

Fedak G, Chen Q, Conner RL, Laroche A, Petroski R and Armstrong KW (2000) Characterization of wheatThinopyrum partial amphiploids by meiotic analysis and genomic in situ hybridization. Genome 43:712-719.

Finch RA (1983) Tissue-specific elimination of alternative whole parental genomes in barley hybrid. Chromosoma 88:386393.

Gerlach WL and Bedbrook JR (1979) Cloning and characterization of ribosomal RNA genes from wheat and barley. Nucleic Acids Res 7:1869-1885.

Gerlach WL and Dyer TA (1980) Sequence organization of the repeated units in the nucleus of wheat, which contains $5 \mathrm{~S}$ rDNA genes. Nucleic Acids Res 8:4851-4865.

Guerra MS and Moraes-Fernandes MIB (1977) Somatic instability in the Brazilian semi-dwarf wheat IAS 54. Can J Genet Cytol 19:225-230.

Heslop-Harrison JS and Schwarzacher T (1993) Molecular cytogenetics-biology and applications in plant breeding. In: 
Sumner AT and Chandley AC (eds) Chromosomes Today. v. 11. Chapman \& Hall, London, pp 191-198.

Heslop-Harrison JS, Schwarzacher T, Anamtawat-Jónsson K, Leitch AR, Shi M and Leitch IJ (1991) In situ hybridization with automated chromosomes denaturation. Technique 3:109-116.

Inagaki M and Tahir M (1990) Comparison of haploid production frequencies in wheat varieties crossed with Hordeum bulbosum L. and maize. Jap J Breed 40:209-216.

Laurie DA and Bennett MD (1986) Wheat x maize hybridization. Can J Genet Cytol 28:313-316.

Laurie DA and Bennett MD (1988) Chromosome behaviour in wheat $\mathrm{x}$ maize, wheat $\mathrm{x}$ sorghum and barley $\mathrm{x}$ maize crosses. In: Kew Chromosome Conference III, HMSO, pp 167-177.

Laurie DA and Bennett MD (1989) The timing of chromosome elimination in hexaploid wheat $\mathrm{x}$ maize crosses. Genome 32:953-961.

McIntosh RA, Hart GE, Devos KM, Gale MD and Rogers WJ (1998) Pathogenic disease/pest reaction. In: McIntosh RA, Hart GE, Devos KM, Gale MD and Rogers WJ (eds) Proc $9^{\text {th }}$ Int Wheat Genet Symp, University of Saskatchewan, Saskatoon, v 5, pp 129-139.

McIntyre CL, Pereira S, Moran LB and Appels R (1990) New Secale cereale (rye) DNA derivatives for the detection of rye chromosome segments in wheat. Genome 33:635-640.
Mukai Y, Endo TR and Gill BS (1990) Physical mapping of the 5S rRNA multigene family in common wheat. J Hered 81:290-295.

Mukai Y, Endo TR and Gill BS (1991) Physical mapping of the 18S.26S rRNA multigene family in common wheat: Identification of a new locus. Chromosoma 100:71-78.

Pedersen C and Langridge P (1997) Identification of the entire chromosome complement of bread by two-colour FISH. Genome 40:589-593.

Schlegel R (2003) Rye introgressions. Gatersleben: Plant Breeding Updates. http://www.desicca.de/plant_breeding/Rye_introgressions/wheat-rye_translocation_II/hauptte il_wheat-rye_translocation_ii.html.

Suenaga K and Nakajima K (1989) Efficient production of haploid wheat (Triticum aestivum $\mathrm{L}$.) through crosses between Japanese wheat and maize (Zea mays). Plant Cell Rep 8:263-266.

Zhang X, Dong Y and Wang RR-C (1996) Characterization of genomes and chromosomes in partial amphiploids of the hybrid Triticum aestivum Thinopyrum ponticum by in situ hybridization, isozyme analysis, and RAPD. Genome 39:1062-1071.

Associate Editor: Everaldo Gonçalves de Barros 